\title{
P-0830. Knowledge and behaviors related to diabetes mellitus among adult Russian population: results of the NATION study
}

\author{
Dedov I. ${ }^{a}$, Shestakova M. ${ }^{\mathrm{ab}}$, Galstyan G. ${ }^{\mathrm{ab}}$, Massi Benedetti M c. \\ a Endocrinology Research Centre, Moscow, Russia; b I.M.Sechenov First Moscow State Medical University, \\ Moscow, Russia;c Hub for International Health Research (HIRS), Perugia, Italy
}

\begin{abstract}
Background: Prevalence of diabetes mellitus type 2 (T2DM) is growing worldwide and has reached epidemic proportions in many countries. ${ }^{1}$ The NATION study allowed to get reliable data on T2DM and pre-diabetes prevalence in Russia, and showed that T2DM was undiagnosed in $54 \%$ of people with the disease. ${ }^{2}$ Knowledge on risk factors and appropriate behaviors related to $\mathrm{T} 2 \mathrm{DM}$ are keys to its proper prevention and management.
\end{abstract}

Aim: To assess knowledge on risk and preventive factors as well as health behaviors related to T2DM among adult population of Russia depending on their diabetes status.

Methods: The NATION study is a national, epidemiological, crosssectional study conducted from September 2013 to February 2015 across 8 federal districts and 63 regions. In adults (aged 20-79 years), recruitment was stratified by age, sex, geographic region and settlement type to obtain a representative sample. Recruitment was done in public areas with high numbers of people. T2DM was diagnosed using glycated haemoglobin A1c (HbA1c) centrally measured in whole blood (diabetes: HbA1c $\geq 6.5 \%$; prediabetes: HbA1c $\geq 5.7 \%-<6.5 \%$ ). HbA1c levels were analyzed by capillary electrophoresis (NGSP Certified method) using a Capillarys 2 FlexPiercing instrument (Sebia Inc, Norcross, GA, USA). Socio-demographic, anthropometric, behavioral data and knowledge on DM risk and preventive factors were collected using a semi-structured questionnaire.

\begin{tabular}{|l|l|}
\hline \multicolumn{2}{|c|}{ Categories of questions: } \\
\hline Occupation, job characteristics. & Measures that can prevent DM \\
\hline Economic status & Gestational DM \\
\hline Smoking & Sleep disorders \\
\hline Alcohol consumption & Diabetes risk factors \\
\hline Physical activity & Family history \\
\hline Food and drinks consumption. & $\begin{array}{l}\text { Annual health checks, incl. visits to } \\
\text { endocrinologist }\end{array}$ \\
\hline $\begin{array}{l}\text { Pregnancy and childbirth, incl. giving } \\
\text { birth to a baby weighing more than 4 kg }\end{array}$ & $\begin{array}{l}\text { Chronic diseases, incl. hypertension, } \\
\text { CAD, CKD, CVD, and peripheral } \\
\text { arterial diseases }\end{array}$ \\
\hline
\end{tabular}

Results. I. A total of 26,620 respondents were screened. Overall 5.4\% $(\mathrm{n}=1449)$ subjects were diagnosed with T2DM (previously known: $2.5 \%$, $\mathrm{n}=668$; newly diagnosed: $2.9 \%, \mathrm{n}=781) ; 19.3 \%(\mathrm{n}=5128)$ were pre-diabetic subjects; $75.3 \%(\mathrm{n}=20043)$ had normal HbA1c level.

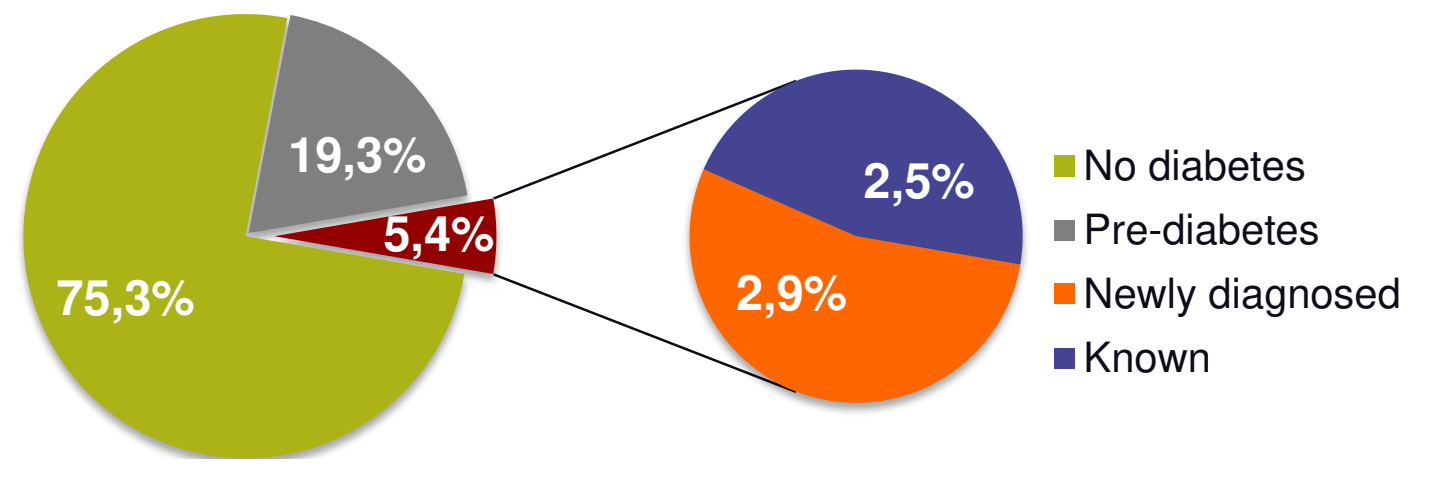

II. Differences were found in knowledge about T2DM risk factors and possible preventive measures between healthy participants and people with previously known diabetes.

\section{Knowledge about T2DM risk factors}

Without diabetes $(n=20043)$
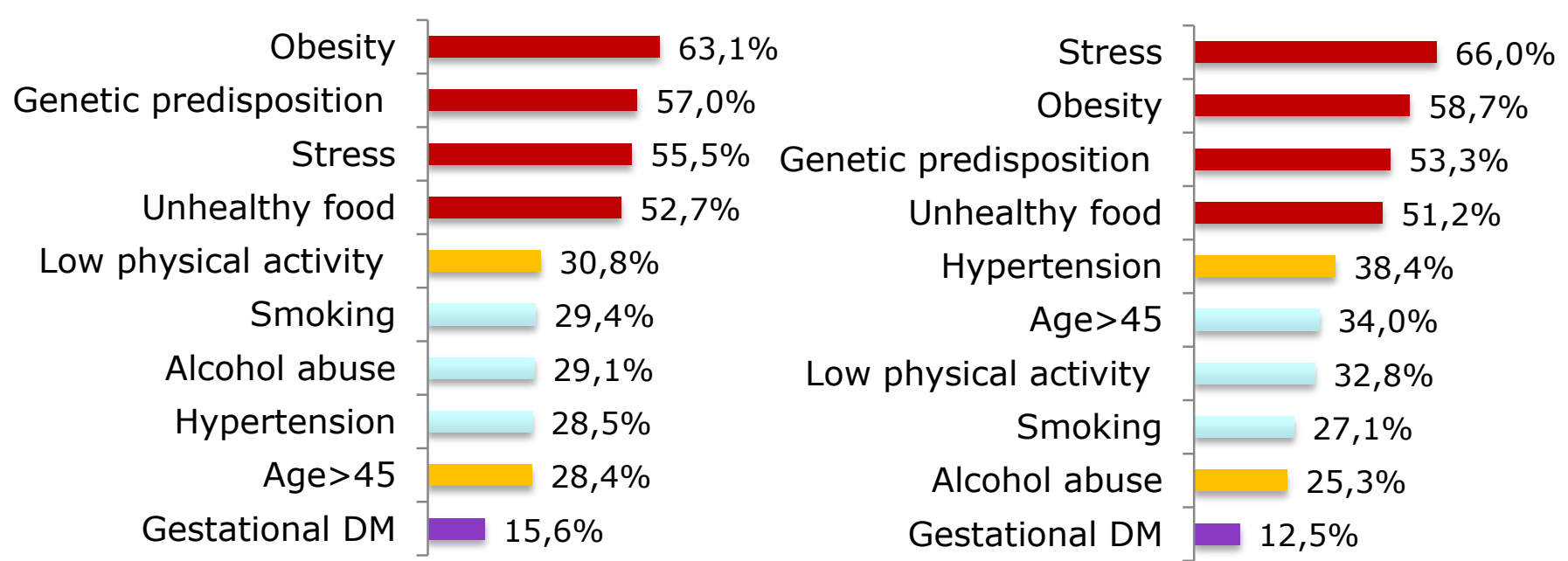

Most frequently participants with previously known T2DM and subjects without diabetes reported the following as risk factors for DM: overweight or obesity $(58.7 \%$ in the group of previously known T2DM and $63.1 \%$ in the group without diabetes, $\mathrm{p}=0.002)$, stress $(66.0 \%$ and $55.5 \%, \mathrm{p}<0.001)$, genetic predisposition $(53.3 \%$ and $57.0 \%, \mathrm{~ns})$ and unhealthy diet $(51.2 \%$ and $52.7 \%$, ns). While overweight or obesity was ranked first by people without diabetes, it was mentioned second after stress by people with previously diagnosed diabetes. About $1 / 3$ of the participants perceived low physical activity as a risk factor for DM (32.8\% in previously known T2DM group and $30.8 \%$ in healthy group). Knowledge of gestational diabetes as a risk factor of DM was low (12.5\% and 15.6\% people with diagnosed T2DM and without disease, respectively).

III. Top-5 measures that can prevent diabetes reported by study participants included the same factors in both groups with previously known T2DM and without diabetes, such as: eating regimen $(66.6 \%$ and $57.3 \%)$, regular physical activity (63.9\% and 56.7\%), weight control (61.6\% and 55.6\%), consumption of fruits and vegetables (61.4\% and 55.2\%), consumption of low fat food (56.0\% and $46.7 \%)$. Interestingly, overall proportions of people who knew these factors were bigger among group with previously known diabetes vs people without diabetes ( $\mathrm{p}<0.01$ for all top-5 factors).

\section{Measures that can prevent diabetes}

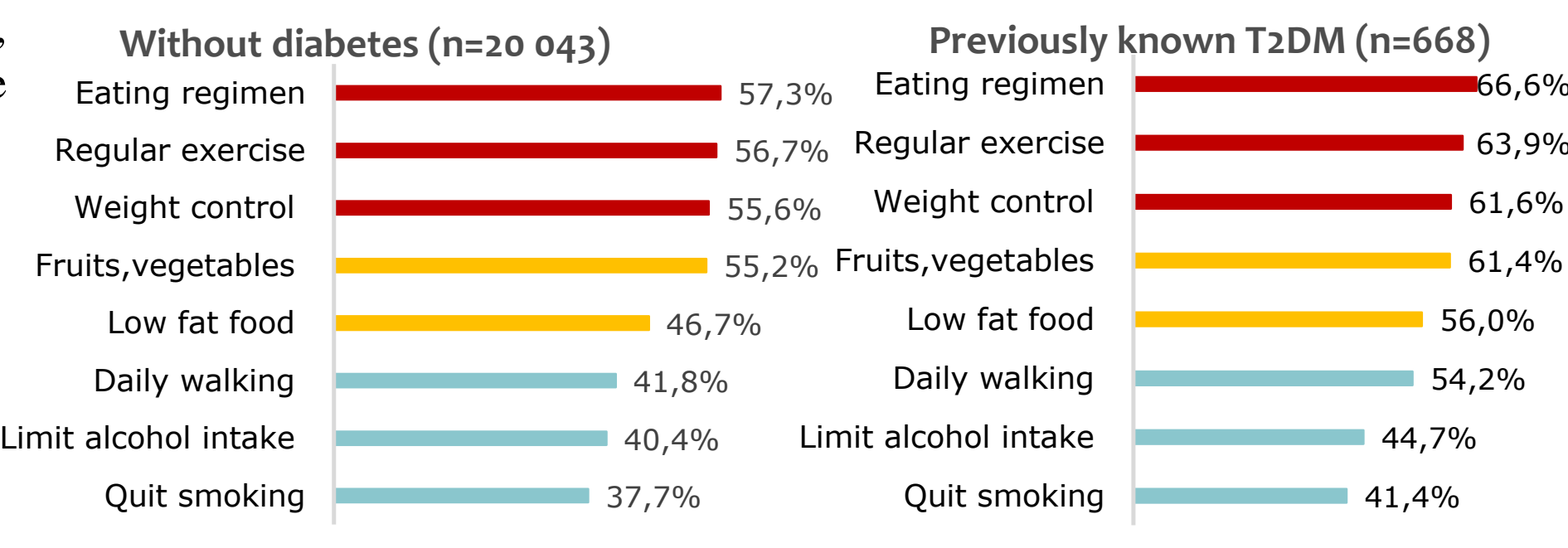

IV. Analysis of health behavior of study participants with previously known T2DM and without diabetes showed high prevalence of low physical activity $(81.6 \%$ and $78.5 \%)$. Only $2 / 3$ of participants eat fruits and vegetables every day, with greater proportion of people with diabetes eating fruits and vegetables every day vs healthy adults $(66.7 \%$ vs $60.0 \%, \mathrm{n}<0.001)$. Proportion of smokers was lower in previously known T2DM group than among people without diabetes ( $14.3 \%$ vs $30.6 \%, \mathrm{n}<0.001)$.

\section{Health behaviors}
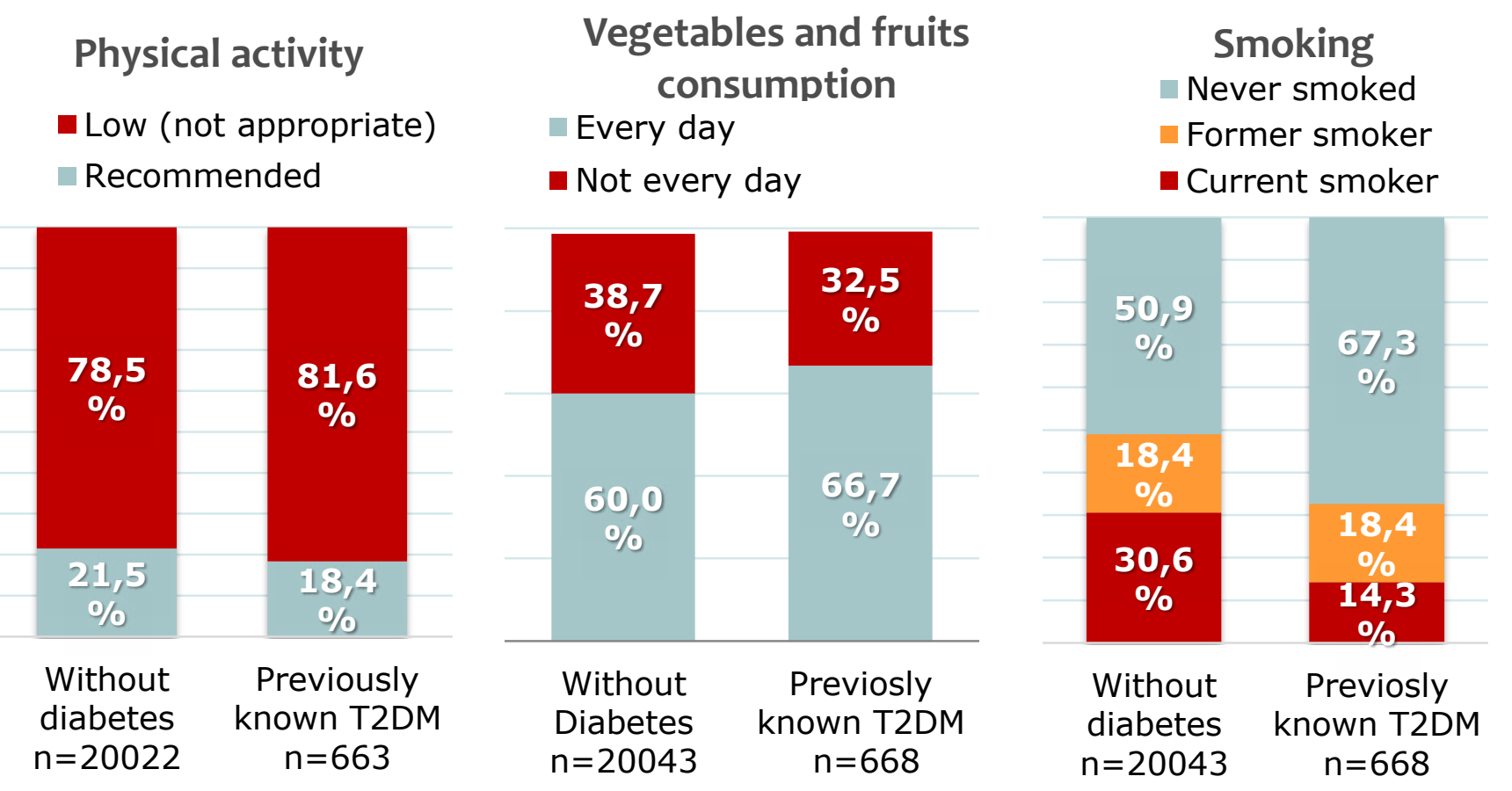

Conclusion: Despite rather good knowledge about the main diabetes risk and preventive factors, the majority of Russian adult population, similarly people with previously known diabetes and without T2DM, demonstrate unhealthy diabetes-related behaviors. These data emphasize the need to implement in Russia a life style intervention program in high risk population as a preventive measure for $\mathrm{T} 2 \mathrm{DM}$, and promotion of early treatment and education programs for people with T2DM.

References

1. IDF Atlas ( $7^{\text {th }}$ edition update). Brussels, Belgium. International Diabetes Federation. Available a http://www.diabetesatlas.org; 2015 [last accessed 01.11.2017]

I.Dedov, M. Shestakova, M.M. Benedetti, D. Simon, I. Pakhomov, G. Galstyan, Prevalence of Type 2 diabetes mellitus (T2DM) in the adult Russian population (NATION study), Diabetes Research and Clinical Practice 2016; 115:90-95.

Acknowledgments and disclosures: The study was funded by Sanofi. ID declares no conflicts of interest. MS declares speaker honorarium from Novo Nordisk, Sanofi-Aventis, Novartis, Merck Sharp \& Dohme AstraZeneca and Servier, and advisory panels for Novo Nordisk and AstraZeneca. GG has participated in advisory panels for Merck Sharp \& Dohme, AstraZeneca and Novo Nordisk, in speaker bureaus for Eli Lilly, Novo Nordisk, Sanofi, Novartis, Berlin Chemie, and Merck Sharp \& Dohme, and has received research support from BC Pharma and Novartis. MMB declares consultancy honorarium from Sanofi-Aventis, membership of the Novo Nordisk DAWN2 Study IPPC, and speaker honorarium from Nextlevel. 